(A) Check for updates

Cite this: Nanoscale, 2020, 12, 11908

\section{Hybrid phase 1T/2H-MoS 2 with controllable 1T concentration and its promoted hydrogen evolution reaction $\uparrow$}

\author{
Yuxiao Zhang, (DD a Yasutaka Kuwahara, (D) ${ }^{a, b, c}$ Kohsuke Mori, (D) ${ }^{a, b}$ Catherine Louis $^{d}$ \\ and Hiromi Yamashita (D) *a,b
}

\begin{abstract}
$\mathrm{MoS}_{2}$ has been investigated as a low-cost alternative to $\mathrm{Pt}$ in the electrochemical hydrogen evolution reaction. One of the promising methods to further activate $\mathrm{MoS}_{2}$ is phase engineering. $\mathrm{MoS}_{2}$ generally exhibits two kinds of crystalline phases: hexagonal $2 \mathrm{H}$ phase and octahedral $1 \mathrm{~T}$ phase. $1 \mathrm{~T}-\mathrm{MoS}_{2}$ exhibits much better chemical/physical properties than natural semiconductor $2 \mathrm{H}-\mathrm{MoS}_{2}$. However, $1 \mathrm{~T}-\mathrm{MoS}_{2}$ is metastable and its synthesis is still a challenge. Hybrid $1 \mathrm{~T} / 2 \mathrm{H}-\mathrm{MoS}_{2}$ has been synthesized under relatively mild conditions, but controlling the $1 \mathrm{~T} / 2 \mathrm{H}$ ratio is still an issue which has not been discussed in detail. In this study, the synthesis methods of hybrid phase $1 \mathrm{~T} / 2 \mathrm{H}-\mathrm{MoS}_{2}$ with controllable $1 \mathrm{~T}$ concentration are investigated. The electrochemical hydrogen evolution reaction is then evaluated for $1 \mathrm{~T} / 2 \mathrm{H}-\mathrm{MoS}_{2}$ with different $1 \mathrm{~T}$ concentrations by performing both experiments and theoretical calculations.
\end{abstract}

Received 30th March 2020, Accepted 6th May 2020

DOI: $10.1039 / \mathrm{d} 0 \mathrm{nr} 02525 \mathrm{a}$ rsc.li/nanoscale
$2 \mathrm{H}-\mathrm{MoS}_{2}$, the number of catalytically active sites in $\mathrm{MoS}_{2}$ could be increased efficiently while maintaining the stability.

A promising application of $1 \mathrm{~T} / 2 \mathrm{H}-\mathrm{MoS}_{2}$ is the electrochemical hydrogen evolution reaction (HER). To meet the growing demand for renewable energy, $\mathrm{H}_{2}$ has been regarded as an essential fuel candidate in the near future. ${ }^{14,15}$ Though the most active catalyst in the electrochemical HER in acidic media is Pt or Pt alloys, ${ }^{16,17}$ a cheaper substitute candidate is needed when considering the cost and scarcity of Pt. $\mathrm{MoS}_{2}$ has been researched as a promising candidate for HER catalyst, especially $1 \mathrm{~T}-\mathrm{MoS}_{2}$ which is active on both the basal and edge planes (natural $2 \mathrm{H}-\mathrm{MoS}_{2}$ is only active on the edge plane). ${ }^{10,18-20}$ But the high synthesis cost and poor stability of $1 \mathrm{~T}-\mathrm{MoS}_{2}$ make it burdensome to use as a low-cost alternative to Pt. For this reason, $1 \mathrm{~T} / 2 \mathrm{H}-\mathrm{MoS}_{2}$ has more potential for practical application due to its simple synthesis conditions and much better HER performance than $2 \mathrm{H}-\mathrm{MoS}_{2} \cdot{ }^{3,21,22}$

Latest studies have found that by inserting guest ions into $\mathrm{MoS}_{2}$ 1T/2H-MoS${ }_{2}$ could be synthesized. Wang et al. ${ }^{23}$ and Huang et $a .^{24}$ have researched the crystalline structure distortion mechanism by inserting $\mathrm{Li}^{+}$or $\mathrm{Na}^{+}$ions. The charge transfers between the injected electron and $\mathrm{Mo}^{4+}$ ions result in $S$ plane sliding and finally lead to a phase transition. Based on the above mechanism, researchers have further investigated that under milder synthesis conditions, $\mathrm{NH}_{4}{ }^{+}$could permeate into $\mathrm{MoS}_{2}$ instead of $\mathrm{Li}^{+}$or $\mathrm{Na}^{+3,25-27}$ Using an ammoniumcontaining precursor through a hydrothermal process, $\mathrm{NH}_{4}{ }^{+}$ could be easily inserted into $\mathrm{MoS}_{2}$, thereby creating 1T/ $2 \mathrm{H}-\mathrm{MoS}_{2}$. 
Up to now, several methods have been developed to obtain $1 \mathrm{~T} / 2 \mathrm{H}-\mathrm{MoS}_{2}$ usually at a fixed $1 \mathrm{~T}$ concentration. ${ }^{26-29}$ Research on controlling and optimizing the $1 \mathrm{~T} / 2 \mathrm{H}$ ratio in the HER reaction is still unreported. In this work, we discuss the effects of different precursors used in the synthesis of hybrid phase $\mathrm{MoS}_{2}$ containing different concentrations of $1 \mathrm{~T}$ phase. Their HER performances are then tested. In order to further understand the relationship between HER performances and 1T concentration, we have carried out a series of DFT calculations to clarify the different activities between $1 \mathrm{~T}-\mathrm{MoS}_{2}$ and $2 \mathrm{H}-\mathrm{MoS}_{2}$ and for the first time, we have investigated the nature of $1 \mathrm{~T} / 2 \mathrm{H}$ interfacial reactions.

\section{Experimental}

\section{Chemicals}

$\left(\mathrm{NH}_{4}\right)_{6} \mathrm{Mo}_{7} \mathrm{O}_{24} \cdot 6 \mathrm{H}_{2} \mathrm{O}$ (ammonium molybdate tetrahydrate), $\mathrm{NaMoO}_{4} \cdot 2 \mathrm{H}_{2} \mathrm{O}$ (sodium molybdate dihydrate), $\mathrm{CH}_{3} \mathrm{CSNH}_{2}$ (thioacetamide, TAA), N,N-dimethylformamide (DMF), $\mathrm{NH}_{4} \mathrm{HCO}_{3}$ (ammonium bicarbonate) and $\mathrm{H}_{2} \mathrm{SO}_{4}$ (sulphuric acid, 98\%) were purchased from Nacalai Tesque Inc. Nafion 117 solution and $10 \% \mathrm{Pt}$ on carbon were purchased from Sigma-Aldrich Co. Commercial $\mathrm{MoS}_{2}$ was purchased from Alfa Aesar Co. Ltd. All chemical reagents were used without any further purification.

\section{Catalyst synthesis}

Hybrid phase $\mathrm{MoS}_{2}$ was synthesized by a solvothermal method using precursors with different $\mathrm{NH}_{4}{ }^{+}$contents. In this work, we discuss the effects of solvent $\left(\mathrm{H}_{2} \mathrm{O}\right.$ or DMF) and guest ion agent $\left(\mathrm{NH}_{4} \mathrm{HCO}_{3}\right)$.

Synthesis of $\mathbf{M o S}_{2}$. In a typical synthesis process, $0.0028 \mathrm{mmol}$ sodium molybdate was mixed with $0.025 \mathrm{mmol}$ TAA and dissolved in $40 \mathrm{~mL}$ of distilled water, and then the solution was transferred to a $50 \mathrm{~mL}$ Teflon-lined stainless steel autoclave and placed in an oven. The hydrothermal reaction was carried out for $12 \mathrm{~h}$ at $200{ }^{\circ} \mathrm{C}$. The black products were then collected by centrifugation and washed with water and ethanol. The sample was named $\mathrm{MoS}_{2}$.

Synthesis of $1 \mathrm{~T} / \mathbf{2 H}-\mathrm{MoS}_{2}$ (DMF). In a typical synthesis process, $0.02 \mathrm{mmol}$ ammonium molybdate and $0.025 \mathrm{~mol}$ TAA were mixed and then dissolved in an aqueous solution containing different DMF contents (25\%, 50\%, 75\%, and 100\%). The solution was then stirred for $15 \mathrm{~min}$ and transferred to a $50 \mathrm{~mL}$ Teflon-lined stainless steel autoclave and placed in an oven. The solvothermal reaction was carried out for $12 \mathrm{~h}$ at $200{ }^{\circ} \mathrm{C}$. The black products were then collected by centrifugation and washed with water and ethanol. The samples were named $1 \mathrm{~T} / 2 \mathrm{H}-\mathrm{MoS}_{2}$ (D25), 1T/2H-MoS (D50), 1T/2H-MoS (D75) and 1T/2H-MoS 2 (D100), respectively.

Synthesis of $1 \mathrm{~T} / 2 \mathbf{H}-\mathrm{MoS}_{2}\left(\mathbf{N H}_{\mathbf{4}}{ }^{+}\right)$. In a typical synthesis process, $0.02 \mathrm{mmol}$ ammonium molybdate and different amounts (50 mg, $100 \mathrm{mg}, 200 \mathrm{mg}, 400 \mathrm{mg}, 800 \mathrm{mg}$, and $1600 \mathrm{mg}$ ) of guest ion agent (ammonium bicarbonate) were mixed and dissolved in $20 \mathrm{~mL}$ of distilled water. The solution was then stirred for 15 min. 0.025 mol TAA was dissolved in $20 \mathrm{~mL}$ of distilled water and stirred for 15 min separately. After that, these two solutions were mixed and stirred for another $5 \mathrm{~min}$ and then transferred to a $50 \mathrm{~mL}$ Teflon-lined stainless steel autoclave and placed in an oven. The hydrothermal reaction was carried out for $12 \mathrm{~h}$ at $200{ }^{\circ} \mathrm{C}$. The black products were then collected by centrifugation and washed with water and ethanol. The samples were named $1 \mathrm{~T} / 2 \mathrm{H}-\mathrm{MoS}_{2}(\mathrm{~N} x)$, where $x$ = the mass of ammonium bicarbonate (mg).

\section{Characterization}

Thermogravimetric (TG) analysis was performed using a Bruker TG-DTA2010SA system from room temperature to $800{ }^{\circ} \mathrm{C}$ at a heating rate of $10{ }^{\circ} \mathrm{C} \cdot \mathrm{min}^{-1}$. X-ray photoelectron spectroscopy (XPS) spectra were recorded on a Shimadzu ESCA 3200 photoelectron spectrometer using $\mathrm{Mg} \mathrm{K} \alpha$ radiation, and C 1s $(284.6 \mathrm{eV})$ was used as the reference to calibrate the peak position of the element. X-ray diffraction (XRD) spectra were obtained using a Rigaku Ultima IV diffractometer using $\mathrm{Cu} \mathrm{K} \alpha$ radiation ( $\lambda=0.15406 \mathrm{~nm}$ ). Mo K-edge X-ray absorption fine structure (XAFS) measurements were performed in a transmission mode at the beamline 01B1 station with an attached Si(111) monochromator at SPring-8, JASRI, Harima, Japan (prop. no. 2018A1144, 2018A1089). Field emission scanning electron microscopy (FE-SEM) was performed using a JSM-6500F (JEOL Ltd). High-resolution TEM micrographs were obtained with a TEM instrument (Hf-2000, Hitachi) equipped with an energy-dispersive X-ray detector (Kevex) operated at $200 \mathrm{kV}$. The specific surface area $\left(S_{\mathrm{BET}}\right)$ and total pore volume $\left(V_{\text {total }}\right)$ were determined using the $\mathrm{N}_{2}$ adsorption isotherm data obtained at $-196{ }^{\circ} \mathrm{C}$ using a BELSORP-max system (MicrotracBEL Corp.)

\section{Electrochemical measurements}

Electrochemical measurements were performed using an HZ-7000 electrochemical workstation. The hydrogen evolution reactions were evaluated using a three-electrode system in 0.5 $\mathrm{M} \mathrm{H}_{2} \mathrm{SO}_{4}$ with a graphite rod and a saturated $\mathrm{Ag} / \mathrm{AgCl}$ electrode as the counter electrode and the reference electrode, respectively. The linear sweep potential tests run from 0.00 to $-1.20 \mathrm{~V}$ (vs. $\mathrm{Ag} / \mathrm{AgCl}$ ) at a scanning rate of $10 \mathrm{mV} \mathrm{s}^{-1}$ in the three electrode system. The working electrode was prepared on a glassy carbon electrode. The catalyst ink was made by dissolving $5 \mathrm{mg}$ of catalyst in $1 \mathrm{~mL}$ of the water-ethanol (2:1 volume ratio) solvent with $40 \mu \mathrm{L}$ of $5 \%$ Nafion solution. The $\mathrm{Pt} / \mathrm{C}$ ink was made by dissolving $5 \mathrm{mg}$ of $10 \% \mathrm{Pt} / \mathrm{C}$ in $1 \mathrm{~mL}$ of the water-DMF (1:1 volume ratio) solvent with $40 \mu \mathrm{L}$ of $5 \%$ Nafion solution.

\section{DFT calculations}

All DFT calculations were performed using the $\mathrm{Dmol}^{3}$ program in the Materials Studio 17.2 software package using the B3LYP functional combined with the DND basis set. The (100) surface of $2 \mathrm{H}-\mathrm{MoS}_{2}, 1 \mathrm{~T}-\mathrm{MoS}_{2}$ and $1 \mathrm{~T} / 2 \mathrm{H}-\mathrm{MoS}_{2}$ were modeled using a $4 \times 1$ supercell cluster model. The bottom $S$ layer and Mo layer were fixed in their bulk positions, whereas the top layer of $\mathrm{S}$ 
atoms and adsorbates were allowed to relax during geometry optimization.

\section{Results and discussion}

In this work, we have tried to synthesize $\mathrm{MoS}_{2}$ with different $1 \mathrm{~T} / 2 \mathrm{H}$ ratios to achieve a high HER performance in a controllable way. The conception of $1 \mathrm{~T} / 2 \mathrm{H}-\mathrm{MoS}_{2}$ synthesis can be summarized as the insertion of guest ions. ${ }^{26-28}$ Herein, two methods for the synthesis of $1 \mathrm{~T} / 2 \mathrm{H}-\mathrm{MoS}_{2}$ with high $1 \mathrm{~T}$ content are discussed.

\section{Solvothermal process using $N, N$-dimethylformamide (DMF)}

$N, N$-Dimethylformamide with an acyl group could provide $\mathrm{NH}_{4}{ }^{+}$during the solvothermal process, and also its better solubility (than water) could be helpful for the reaction. Firstly, the $1 \mathrm{~T}$ concentration is controlled by using different $\mathrm{DMF} / \mathrm{H}_{2} \mathrm{O}$ solvents in a solvothermal process. Fig. 1 shows the XRD patterns and Mo K-edge FT-EXAFS spectra of the as-prepared samples. The characteristic peaks of $2 \mathrm{H}-\mathrm{MoS}_{2}$ can be observed in the asprepared $\mathrm{MoS}_{2}$ sample, including the (002) $\left(2 \theta=14.4^{\circ}\right),(100)$ $\left(2 \theta=32.7^{\circ}\right),(101)\left(2 \theta=33.5^{\circ}\right),(103)\left(2 \theta=39.6^{\circ}\right)$, and $(110)(2 \theta$ $\left.=58.3^{\circ}\right)$ planes $\left(\mathrm{MoS}_{2}\right.$ card from JCPDS 65-1951). For samples synthesized using DMF, the absence of the (002) and (103) planes, together with a newly-emerged (004) plane at $17.8^{\circ}$ refers to the $1 \mathrm{~T}-\mathrm{MoS}_{2}$ pattern. ${ }^{11,29}$ Besides, a broadening of the (100) and (101) planes, and a shift to the lower angle of the (110) plane could be observed, which correspond to the distortion of the inserted $1 \mathrm{~T}-\mathrm{MoS}_{2}$ crystalline phase. ${ }^{3,27}$ The distortion level of the crystalline structure can be roughly judged by the amount of shift of the (110) plane. From $\mathrm{MoS}_{2}$ to $1 \mathrm{~T} /$ $2 \mathrm{H}-\mathrm{MoS}_{2}$ (D50), the lattice distortion is gradually increased by increasing the amount of DMF. However, $1 \mathrm{~T} / 2 \mathrm{H}-\mathrm{MoS}_{2}$ (D75) and $1 \mathrm{~T} / 2 \mathrm{H}-\mathrm{MoS}_{2}$ (D100) almost exhibit the same shift level as $1 \mathrm{~T} / 2 \mathrm{H}-\mathrm{MoS}_{2}$ (D50). X-ray absorption fine structure (XAFS) spectroscopy also provides evidence for the synthesized $1 \mathrm{~T} /$ $2 \mathrm{H}-\mathrm{MoS}_{2}$. Mo K-edge X-ray absorption near edge structure (XANES) results have been provided in Fig. S1.† Samples synthesized using DMF show a similar absorption edge to $\mathrm{MoS}_{2}$, which indicates that the chemical valence of Mo is +4 . A shoulder peak at $20100 \mathrm{eV}$ can be observed in the $\mathrm{MoS}_{2}$ sample but disappears in samples synthesized using DMF, demonstrating that the lattice structure is changed owing to


Fig. 1 (a) XRD patterns, (b) FT-EXAFS spectra of 1T/2H-MoS 2 (D25), 1T/ $2 \mathrm{H}-\mathrm{MoS}_{2}$ (D50), 1T/2H-MoS (D75), 1T/2H-MoS (D100) and MoS . the inserted $1 \mathrm{~T}$ phase..$^{3,27,30-32}$ To investigate the detail of distortion, Fourier-transforms of extended X-ray absorption fine structure (FT-EXAFS) spectra are obtained (Fig. 1b). The FT magnitude of the nearest coordination sphere of the chemically bonded Mo-Mo (peak B) sphere split into two contributions in samples synthesized using DMF, which is due to the formation of a hybrid $1 \mathrm{~T} / 2 \mathrm{H}$ phase structure. ${ }^{30,33}$ It is worth noting that the intensity of peak B2 is gradually decreased by increasing the amount of DMF and finally the peak disappears in 1T/2H-MoS 2 (D75) and 1T/2H-MoS 2 (D100). Also, the intensity of peak A (corresponding to Mo-S) decreases, and the shape of peak A becomes wider by increasing the amount of DMF. These facts indicate that the crystalline structure would be destroyed by the excessive (more than 75\%) DMF precursor. The TG/DTA is measured under air flow and the measured data are shown in Fig. S2. $\dagger$ The $\mathrm{MoS}_{2}$ sample showed only one endothermic peak with a mass loss at around $420{ }^{\circ} \mathrm{C}$, which corresponds to the oxidation of $\mathrm{MoS}_{2} \cdot{ }^{25}$ Different from $\mathrm{MoS}_{2}, 1 \mathrm{~T} / 2 \mathrm{H}-\mathrm{MoS}_{2}$ (D25) and 1T/2H-MoS $(\mathrm{D} 50)$ samples showed a mass loss at around $200{ }^{\circ} \mathrm{C}$ with two DTA peaks at around $230{ }^{\circ} \mathrm{C}$ and $250{ }^{\circ} \mathrm{C}$, which represent the deintercalation of $\mathrm{NH}_{4}^{+}$and a phase change from $1 \mathrm{~T}$ to $2 \mathrm{H}$, respectively. ${ }^{3,26,27}$ It should be noted that $1 \mathrm{~T} / 2 \mathrm{H}-\mathrm{MoS}_{2}$ (D75) and $1 \mathrm{~T} / 2 \mathrm{H}-\mathrm{MoS}_{2}$ (D100) exhibited only one endothermic peak around $230-250{ }^{\circ} \mathrm{C}$, possibly due to the destroyed crystal lattice structure as shown in the FT-EXAFS spectra. The hybridized $1 \mathrm{~T} / 2 \mathrm{H}$ structure could also be identified in Mo $3 \mathrm{~d}$ and $\mathrm{S}$ 2 p XPS spectra as shown in Fig. 2 and Fig. S3. $\dagger$ In the Mo $3 \mathrm{~d}$ spectrum of $\mathrm{MoS}_{2}$, resonance at $229.3 \mathrm{eV}$ and $232.2 \mathrm{eV}$ correspond to typical $3 \mathrm{~d}_{5 / 2}$ and $3 \mathrm{~d}_{3 / 2}$ peaks of $2 \mathrm{H}-\mathrm{MoS}_{2}$, respectively. Nevertheless, two new peaks emerged at lower binding energy in samples synthesized using DMF. The new peaks at around

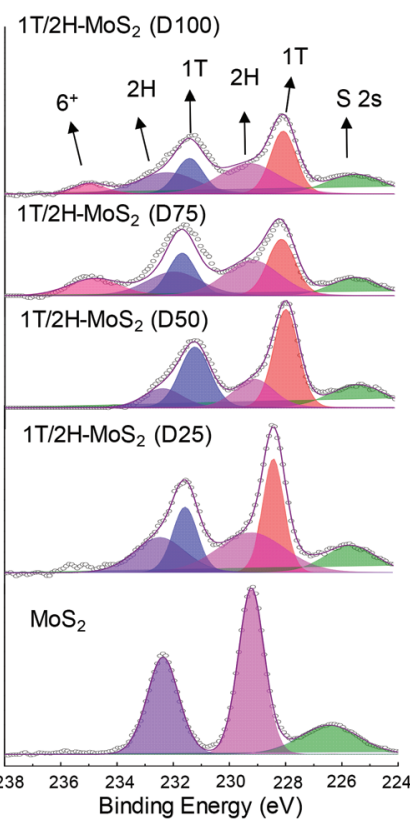

Fig. 2 Mo 3d XPS spectra of 1T/2H-MoS (D25), 1T/2H-MoS 2 (D50), 1T/ $2 \mathrm{H}-\mathrm{MoS}_{2}$ (D75), 1T/2H-MoS 2 (D100) and $\mathrm{MoS}_{2}$ 
$228.2 \mathrm{eV}$ and $231.3 \mathrm{eV}$ correspond to $1 \mathrm{~T}-\mathrm{MoS}_{2}$ in the hybrid $1 \mathrm{~T} /$ $2 \mathrm{H}$ structure. ${ }^{33-37}$ In addition, the $\mathrm{Mo}^{6+} 3 \mathrm{~d}_{5 / 2}$ peak appeared in $1 \mathrm{~T} / 2 \mathrm{H}-\mathrm{MoS}_{2}$ (D75) and 1T/2H-MoS $(\mathrm{D} 100)$ spectra, indicating that $\mathrm{MoS}_{2}$ is partly oxidized under these conditions. Furthermore, the $\mathrm{Mo}^{6+}$ peak ratio is lower in $1 \mathrm{~T} / 2 \mathrm{H}-\mathrm{MoS}_{2}$ (D100) than in $1 \mathrm{~T} / 2 \mathrm{H}-\mathrm{MoS}_{2}$ (D75), which is because of the reducibility of DMF. S 2p spectra also show similar results to Mo 3d spectra. The existence of the $1 \mathrm{~T} / 2 \mathrm{H}$ hybrid structure could be directly observed by high-resolution TEM (HR-TEM). The HR-TEM image of $1 \mathrm{~T} / 2 \mathrm{H}-\mathrm{MoS}_{2}$ is shown in Fig. 3(a) and its fast Fourier transform pattern is shown in Fig. 3(b). To identify the positions of the existing different lattice structures, the inverse Fourier transform images that emphasized the periodicity of the (110) plane were obtained at different frequencies, as shown in Fig. S4(c) and (d). $\dagger$ From these distribution images, area 1 and area 2 were chosen for further analysis. The hexagonal lattice region could be observed clearly in area 1 with a crystalline space of $0.27 \mathrm{~nm}$, which is ascribed to the (100) plane of $2 \mathrm{H}-\mathrm{MoS}_{2}$ (Fig. 3(c) and (e)). Otherwise, the trigonal lattice region in area 2 that showed a crystalline space of $0.24 \mathrm{~nm}$ is indexed to the (100) plane of $1 \mathrm{~T}-\mathrm{MoS}_{2}$ (Fig. 3(d) and (f)). In addition, to show the combination structure of the $1 \mathrm{~T} / 2 \mathrm{H}$ hybrid phase, dark-field images focused on the lattice space of $0.24 \mathrm{~nm}$ and $0.27 \mathrm{~nm}$ were obtained, as shown in Fig. S5. $\dagger$ The dark-field images were colored and stacked together so that the mixed state could be intuitively observed.

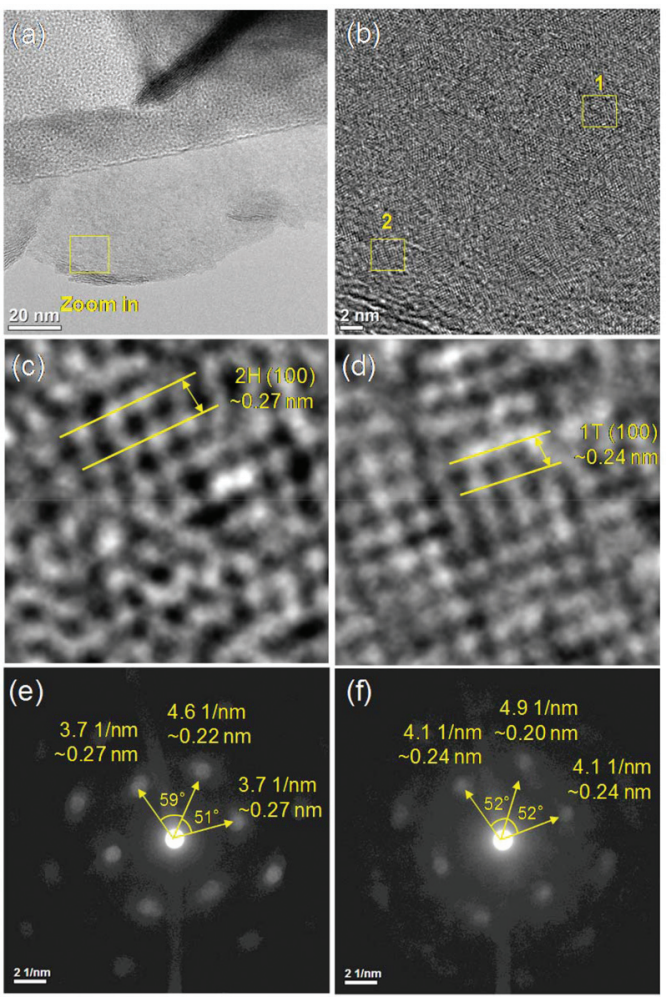

Fig. 3 (a), (b) High-resolution TEM bright field images of $1 \mathrm{~T} / 2 \mathrm{H}-\mathrm{MoS}_{2}$, (c), (d) zoom in of area 1 and 2 respectively, and (e), (f) selected area electron diffraction (SAED) of area 1 and 2, respectively.
Table 1 The 1T/2H concentration of samples synthesized using different amounts of DMF

\begin{tabular}{lll}
\hline Sample & $1 \mathrm{~T}(\%)$ & $2 \mathrm{H}(\%)$ \\
\hline $\mathrm{MoS}_{2}$ & - & 100 \\
$1 \mathrm{~T} / 2 \mathrm{H}-\mathrm{MoS}_{2}(\mathrm{D} 25)$ & 47.99 & 52.01 \\
$1 \mathrm{~T} / 2 \mathrm{H}-\mathrm{MoS}_{2}$ (D50) & 70.14 & 29.86 \\
$1 \mathrm{~T} / 2 \mathrm{H}-\mathrm{MoS}_{2}$ (D75) & 46.03 & 53.97 \\
$1 \mathrm{~T} / 2 \mathrm{H}-\mathrm{MoS}_{2}$ (D100) & 45.83 & 54.17
\end{tabular}

From these pieces of evidence, the hybrid $1 \mathrm{~T} / 2 \mathrm{H}$ phase in $\mathrm{MoS}_{2}$ prepared using DMF was identified.

In order to quantify the concentrations of $1 \mathrm{~T}-\mathrm{MoS}_{2}$ in a hybrid sample, the multi-peak curve fitting of XPS spectra was carried out. The $1 \mathrm{~T}$ and $2 \mathrm{H}$ concentration of samples are presented in Table 1. Among these samples, 1T/2H-MoS 2 (D50) exhibited the highest $1 \mathrm{~T}$ concentration, which was about $70.13 \%$ of $1 \mathrm{~T}-\mathrm{MoS}_{2}$. With an increase in the DMF amount, the 1T percentage initially increases and then decreases. The increase in the $1 \mathrm{~T}$ concentration is consistent with the peak shift of the (110) plane in XRD and higher intensity ratio of peak B1:B2 in FT-EXAFS. Otherwise, the decrease in the $1 \mathrm{~T}: 2 \mathrm{H}$ ratio could be attributed to the destruction of the crystalline structure and oxidation, as discussed above. In order to further clarify the reason, a series of SEM images and $\mathrm{N}_{2}$ absorption-desorption isotherms have been obtained. From SEM images shown in Fig. 4, an open flower-like structure could be observed in the $\mathrm{MoS}_{2}, 1 \mathrm{~T} / 2 \mathrm{H}-\mathrm{MoS}_{2}$ (D25) and 1T/ $2 \mathrm{H}-\mathrm{MoS}_{2}$ (D50) samples. These structures usually have large surface areas, as shown in Fig. S6 and Table $\mathrm{S} 1 \uparrow\left(S_{\mathrm{BET}}=\right.$ $55.2 \mathrm{~m}^{2} \mathrm{~g}^{-1}, 51.8 \mathrm{~m}^{2} \mathrm{~g}^{-1}$ and $31.6 \mathrm{~m}^{2} \mathrm{~g}^{-1}$, respectively), which facilitate electron transfer between the catalyst and the reaction compound. ${ }^{3,5}$ However, nonideal flake-like structures are observed in the $1 \mathrm{~T} / 2 \mathrm{H}-\mathrm{MoS}_{2}$ (D75) sample, which result in a lower surface area $\left(S_{\mathrm{BET}}=10.6 \mathrm{~m}^{2} \mathrm{~g}^{-1}\right)$. Besides, only a few flake-like structures appear in $1 \mathrm{~T} / 2 \mathrm{H}-\mathrm{MoS}_{2}$ (D100), and hence this sample exhibits higher surface area $\left(S_{\mathrm{BET}}=28.3 \mathrm{~m}^{2} \mathrm{~g}^{-1}\right)$ than $1 \mathrm{~T} / 2 \mathrm{H}-\mathrm{MoS}_{2}$ (D75). Combining with the XPS data, the flake-like structures may originate from the oxidized molybdenum. In addition, compared with the open structures in Fig. 4(b) and (c), the surface structure of $1 \mathrm{~T} / 2 \mathrm{H}-\mathrm{MoS}_{2}$ (D100)

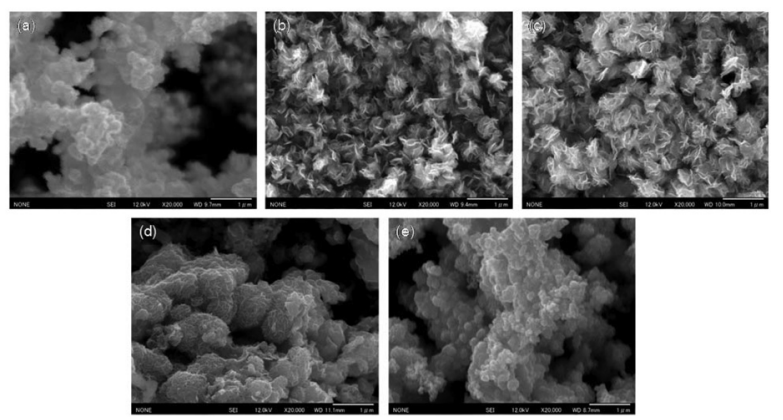

Fig. 4 FE-SEM images of (a) $\mathrm{MoS}_{2}$, (b) $1 \mathrm{~T} / 2 \mathrm{H}-\mathrm{MoS}_{2}$ (D25), (c) 1T/ $2 \mathrm{H}-\mathrm{MoS}_{2}$ (D50), (d) $1 \mathrm{~T} / 2 \mathrm{H}-\mathrm{MoS}_{2}$ (D75) and (e) $1 \mathrm{~T} / 2 \mathrm{H}-\mathrm{MoS}_{2}$ (D100). 
(Fig. 4(e)) seems closed due to the different solvent conditions. ${ }^{4}$ The destruction of the structure results in lower surface area $\left(S_{\mathrm{BET}}=28.3 \mathrm{~m}^{2} \mathrm{~g}^{-1}\right)$ and total pore volume $\left(V_{\text {total }}=\right.$ $\left.0.099 \mathrm{~cm}^{3} \mathrm{~g}^{-1}\right)$ than $1 \mathrm{~T} / 2 \mathrm{H}-\mathrm{MoS}_{2}$ (D50) $\left(S_{\text {BET }}=31.6 \mathrm{~m}^{2} \mathrm{~g}^{-1}\right.$ and $\left.V_{\text {total }}=0.146 \mathrm{~cm}^{3} \mathrm{~g}^{-1}\right)$.

The electrocatalytic HER performance of the $\mathrm{MoS}_{2}$ sample is tested under acidic conditions $\left(0.5 \mathrm{M} \mathrm{H}_{2} \mathrm{SO}_{4}\right)$ using a threeelectrode system by LSV method at a scan rate of $10 \mathrm{mV} \mathrm{s}^{-1}$. The LSV curves are shown in Fig. 5, and the onset overpotential and Tafel slope values are listed in Table S2. $\dagger$ Former research studies on the HER have reported that $2 \mathrm{H}-\mathrm{MoS}_{2}$ is only active on the edge plane, while $1 \mathrm{~T}-\mathrm{MoS}_{2}$ is active on both the basal and edge planes. ${ }^{2,18}$ For this reason, the intrinsic activity of $1 \mathrm{~T} / 2 \mathrm{H}-\mathrm{MoS}_{2}$ is predicted to depend on the $1 \mathrm{~T}$ concentration. From Fig. 5(a), it can be observed that the $1 \mathrm{~T} /$ $2 \mathrm{H}-\mathrm{MoS}_{2}$ (D50) sample exhibited the best HER activity with the lowest onset overpotential of $146.6 \mathrm{mV}$ which is closer to that of the Pt/C reference electrode (onset overpotential: $45.5 \mathrm{mV}$ ). Among these samples, $\mathrm{MoS}_{2}$ exhibited a high onset overpotential of $270.5 \mathrm{mV}$. 1T/2H-MoS 2 (D25) exhibited an onset overpotential of $243.6 \mathrm{mV}$, and $1 \mathrm{~T} / 2 \mathrm{H}-\mathrm{MoS}_{2}$ (D75) and $1 \mathrm{~T} / 2 \mathrm{H}-\mathrm{MoS}_{2}$ (D100) exhibited onset overpotentials of $226.2 \mathrm{mV}$ and $240.2 \mathrm{mV}$, respectively. The $1 \mathrm{~T}$ percentages and onset overpotential values are shown in Fig. 5(b). From this figure, a good positive correlation between HER activities and 1T concentrations could be observed. The specific activity normalized by the BET surface area could also be used to investigate the intrinsic activity. The specific activity is defined as $j / S_{\mathrm{r}}(\mathrm{mA}$ $\mathrm{cm}^{-2}$ ), where $S_{\mathrm{r}}$ is accessible/electrochemical active surface area of the catalyst (obtained from the BET surface area) and $j$ is the HER current. Fig. S7† shows the specific activity at $-0.35 \mathrm{~V}$. The $1 \mathrm{~T} / 2 \mathrm{H}-\mathrm{MoS}_{2}$ (D50) sample shows the highest specific activity at this potential. Although $1 \mathrm{~T} / 2 \mathrm{H}-\mathrm{MoS}_{2}(\mathrm{D} 25)$ and $1 \mathrm{~T} /$
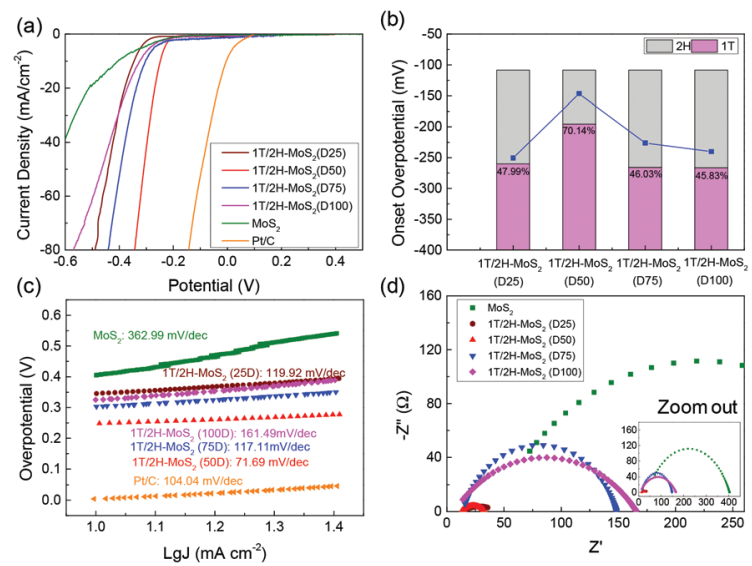

Fig. 5 (a) Linear sweep voltammetry (LSV) curves of $1 \mathrm{~T} / 2 \mathrm{H}-\mathrm{MoS}_{2}$ (D25), $1 \mathrm{~T} / 2 \mathrm{H}-\mathrm{MoS}_{2}$ (D50), 1T/2H-MoS 2 (D75), 1T/2H-MoS 2 (D100), MoS 2 , and $\mathrm{Pt} / \mathrm{C}$. (b) The relevant relation between onset overpotential and $1 \mathrm{~T}$ concentration. (c) Tafel slopes of $1 \mathrm{~T} / 2 \mathrm{H}-\mathrm{MoS}_{2}$ (D25), 1T/2H-MoS 2 (D50), 1T/ $2 \mathrm{H}-\mathrm{MoS}_{2}$ (D75), 1T/2H-MoS $(\mathrm{D} 100), \mathrm{MoS}_{2}$, and Pt/C. (d) Nyquist plots of $1 \mathrm{~T} / 2 \mathrm{H}-\mathrm{MoS}_{2}$ (D25), 1T/2H-MoS (D50), 1T/2H-MoS (D75), 1T/2H-MoS 2 (D100) and $\mathrm{MoS}_{2}$.
$2 \mathrm{H}-\mathrm{MoS}_{2}$ (D75) exhibit similar $1 \mathrm{~T}$ concentrations, the difference between their surface morphologies leads to different specific activities, which indicates that the morphology also plays an important role in intrinsic activity. Fig. 5(c) displays the Tafel plots of these samples. The Tafel slope is an important indicator of reaction kinetics. The Tafel plots are fit to the Tafel equation $(\eta=b \log j+a$, where $j$ is the current density and $b$ is the Tafel slope) to get the Tafel slopes. Among these samples, $1 \mathrm{~T} / 2 \mathrm{H}-\mathrm{MoS}_{2}$ (D50) exhibited the lowest Tafel slope owing to its faster electron transfer kinetics resulting from a higher content of $1 \mathrm{~T}-\mathrm{MoS}_{2}$. Although $1 \mathrm{~T} / 2 \mathrm{H}-\mathrm{MoS}_{2}$ (D75) shows a similar $1 \mathrm{~T}$ concentration to $1 \mathrm{~T} / 2 \mathrm{H}-\mathrm{MoS}_{2}$ (D100), the HER performance of $1 \mathrm{~T} / 2 \mathrm{H}-\mathrm{MoS}_{2}$ (D75) is slightly higher than that of $1 \mathrm{~T} / 2 \mathrm{H}-\mathrm{MoS}_{2}$ (D100). This may be because the oxidized molybdenum in $1 \mathrm{~T} / 2 \mathrm{H}-\mathrm{MoS}_{2}$ (D75) is devoted to the reaction, or the destroyed crystalline structure in $1 \mathrm{~T} / 2 \mathrm{H}-\mathrm{MoS}_{2}$ (D100) hinders the reaction. Furthermore, the electrochemical impedance spectroscopy results are displayed in Fig. $5(\mathrm{~d})$. The $R_{\mathrm{s}}$ and $R_{\mathrm{ct}}$ values are listed in Table S2. $\dagger$ Among these samples, 1T/ $2 \mathrm{H}-\mathrm{MoS}_{2}$ (D50) showed the smallest semicircle, suggesting its lower charge transfer resistance $\left(R_{\mathrm{ct}}=20.9 \Omega\right)$. The lower $R_{\mathrm{ct}}$ affords faster HER kinetics brought about by the higher content of $1 \mathrm{~T}-\mathrm{MoS}_{2}$, which we will discuss later in the section DFT calculations. The lower $R_{\mathrm{s}}$ of $1 \mathrm{~T} / 2 \mathrm{H}-\mathrm{MoS}_{2}$ samples than $\mathrm{MoS}_{2}$ indicates that the metallic $1 \mathrm{~T}$ phase contributed to the conductivity of catalysts. All the electrochemical results demonstrated that the HER performance and 1T concentration have a positive correlation. The electrochemical stability of $1 \mathrm{~T} /$ $2 \mathrm{H}-\mathrm{MoS}_{2}$ (D50) was obtained using the chronoamperometric curve $(I-t)$ at a static overpotential of $0.3 \mathrm{~V}$, as shown in Fig. S8. $\uparrow$ The current density decreased slightly during 4 hours of running, which indicated its good stability.

\section{Hydrothermal process with $\mathrm{NH}_{4} \mathrm{HCO}_{3}$ as the guest ion agent}

Another method to synthesize $1 \mathrm{~T} / 2 \mathrm{H}-\mathrm{MoS}_{2}$ is to introduce a guest ion agent during a hydrothermal process. Different from creating a $1 \mathrm{~T}$ phase using DMF, the hydrothermal process provides relatively mild conditions that are favorable for crystallinity. In order to control the $1 \mathrm{~T}$ concentration, a series of catalysts are synthesized by varying the amount of guest ion agent. The mass of the guest ion agent is varied from $50 \mathrm{mg}$ (about 5 mass \% of other precursors) to $1600 \mathrm{mg}$ (about 200 mass\% of other precursors). The XRD patterns and HER performances are illustrated in Fig. 6. Firstly, the 1T concentration could be roughly judged by the shift of the (110) plane peak, as we discussed above. With the increasing amount of guest ion agent, the as-prepared samples showed almost the same XRD pattern, with the amount of guest ion agent ranging from $50 \mathrm{mg}$ to $800 \mathrm{mg}$ in the precursor. When the amount of precursor reached $1600 \mathrm{mg}$, the peak of the (110) plane emerged at around $58^{\circ}$, which was close to that of $2 \mathrm{H}-\mathrm{MoS}_{2}$. The HER performances were tested and are shown in Fig. 6(b), and the Tafel slopes are shown in Fig. S9(a). $\dagger$ It is obvious that similar HER activity was exhibited by samples synthesized using different amounts of guest ion agent ranging from $50 \mathrm{mg}$ to $800 \mathrm{mg}$ in the precursor (with an onset overpotential of 
(a)

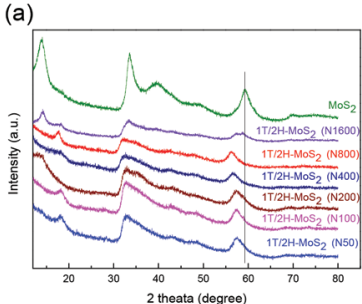

(b)

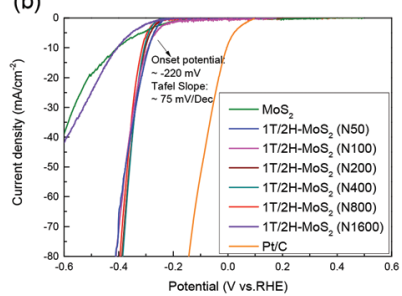

Fig. 6 (a) XRD patterns of $1 \mathrm{~T} / 2 \mathrm{H}-\mathrm{MoS}_{2}$ (N50), 1T/2H-MoS 2 (N100), 1T/ $2 \mathrm{H}-\mathrm{MoS}_{2}$ (N200), 1T/2H-MoS $(\mathrm{N} 400), 1 \mathrm{~T} / 2 \mathrm{H}-\mathrm{MoS}_{2}$ (N800), 1T/2H-MoS (N1600) and $\mathrm{MoS}_{2}$. (b) Linear sweep voltammetry (LSV) curves of $1 \mathrm{~T} /$ $2 \mathrm{H}-\mathrm{MoS}_{2}$ (N50), 1T/2H-MoS $(\mathrm{N} 100), 1 \mathrm{~T} / 2 \mathrm{H}-\mathrm{MoS}_{2}$ (N200), 1T/2H-MoS (N400), 1T/2H-MoS $(\mathrm{N} 800), 1 \mathrm{~T} / 2 \mathrm{H}-\mathrm{MoS}_{2}$ (N1600) $\mathrm{MoS}_{2}$, and Pt/C.

$\sim 220 \mathrm{mV}$ and a Tafel slope of $\sim 75 \mathrm{mV} \mathrm{dec}{ }^{-1}$, as summarized in Table S $3 \uparrow$ ). Corresponding to the XRD patterns, when the precursor amount reached $1600 \mathrm{mg}$, it showed much worse HER performance than the other samples (with an onset overpotential of $264.1 \mathrm{mV}$ and a Tafel slope of $\left.241.9 \mathrm{mV} \mathrm{dec}^{-1}\right)$. It should be noted that the charge transfer resistance shown by Nyquist plots (Fig. S9(b), $\dagger$ the values are listed in Table S3 $\dagger$ ) also follow the same tendency as those observed from the HER and XRD results. The above characterization and catalytic test suggest that by using a guest ion agent in a hydrothermal process, the hybrid $1 \mathrm{~T} / 2 \mathrm{H}$ phase with fixed concentration could be generated easily but difficult to be controlled. On the other hand, 1T/2H-MoS 2 could be stably synthesized with a huge adjustable range of precursor amount. Since the samples showed similar properties, the $1 \mathrm{~T}$ concentration was quantified by $1 \mathrm{~T} / 2 \mathrm{H}-\mathrm{MoS}_{2}$ (N400), $1 \mathrm{~T} / 2 \mathrm{H}-\mathrm{MoS}_{2}$ (N800), and $1 \mathrm{~T} /$ $2 \mathrm{H}-\mathrm{MoS}_{2}$ (N1600). The XPS spectra of Mo 3d and S 2p are shown in Fig. S10 $\dagger$ and the 1T concentrations are presented in Table 2. The samples synthesized with less than $800 \mathrm{mg}$ of guest ion agent exhibited around $60 \% 1 \mathrm{~T}$ concentration. This $1 \mathrm{~T}$ concentration was lower than that of $1 \mathrm{~T} / 2 \mathrm{H}-\mathrm{MoS}_{2}$ (D50) but was higher than those of the other samples synthesized using DMF. The $1 \mathrm{~T} / 2 \mathrm{H}-\mathrm{MoS}_{2}$ (N1600) sample exhibited a small amount of $1 \mathrm{~T}$ content $(26.06 \%)$, maybe due to the existence of excessive ions or improper $\mathrm{pH}$ of the precursor solution. Although this method could not reach extremely high 1T concentration, it is a feasible synthesis process to reach a fixed content of $1 \mathrm{~T}$ phase in $1 \mathrm{~T} / 2 \mathrm{H}-\mathrm{MoS}_{2}$.

\section{DFT calculations}

From the above experimental results, it can be noted that the $1 \mathrm{~T}$ concentration of hybrid $1 \mathrm{~T} / 2 \mathrm{H}-\mathrm{MoS}_{2}$ is positively correlated

Table 2 The $1 \mathrm{~T} / 2 \mathrm{H}$ concentration of samples synthesized using different amounts of $\mathrm{NH}_{4} \mathrm{HCO}_{3}$

\begin{tabular}{lll}
\hline Sample & $1 \mathrm{~T}(\%)$ & $2 \mathrm{H}(\%)$ \\
\hline $\mathrm{MoS}_{2}$ & - & 100 \\
$1 \mathrm{~T} / 2 \mathrm{H}-\mathrm{MoS}_{2}(\mathrm{~N} 400)$ & 60.45 & 39.55 \\
$1 \mathrm{~T} / 2 \mathrm{H}-\mathrm{MoS}_{2}(\mathrm{~N} 800)$ & 58.49 & 41.51 \\
$1 \mathrm{~T} / 2 \mathrm{H}-\mathrm{MoS}_{2}(\mathrm{~N} 1600)$ & 26.06 & 73.94
\end{tabular}

with the HER performance. To better understand the reaction kinetics of $1 \mathrm{~T} / 2 \mathrm{H}-\mathrm{MoS}_{2}$ in the HER process, the nature of the reaction on the $1 \mathrm{~T}-\mathrm{MoS}_{2}, 2 \mathrm{H}-\mathrm{MoS}_{2}$ and $1 \mathrm{~T} / 2 \mathrm{H}$ interface was investigated by DFT calculations. The reaction site was chosen as $\mathrm{S}$ atoms on the (100) surface because it has been reported to be an active site on a typically exposed surface of $\mathrm{MoS}_{2}{ }^{38}$ The HER mechanism under acidic conditions is usually considered as follows:

$$
\begin{gathered}
\mathrm{H}_{3} \mathrm{O}^{+}+\mathrm{e}^{-}+* \rightarrow \mathrm{H} *+\mathrm{H}_{2} \mathrm{O} \text { (Volmer reaction) } \\
\mathrm{H} *+\mathrm{H}_{3} \mathrm{O}^{+}+\mathrm{e}^{-} \rightarrow \mathrm{H}_{2}+\mathrm{H}_{2} \mathrm{O} \text { (Heyrovsky reaction) } \\
\mathrm{H} *+\mathrm{H} * \rightarrow \mathrm{H}_{2} \text { (Tafel reaction) }
\end{gathered}
$$

The initial adsorption of protons from an acidic solution to form adsorbed H (R1) is the first step of the HER process. The following two paths could also be considered. One is the reaction between adsorbed $\mathrm{H}^{*}$ and an extra solvated proton to form $\mathrm{H}_{2}$ (Heyrovsky reaction). Another is the reaction of two adsorbed $\mathrm{H}^{*}$ to form one molecule of $\mathrm{H}_{2}$ (Tafel reaction). In a DFT process, the energy barriers of the Volmer reaction and Heyrovsky reaction are approximated from the energy barriers of analogous chemical reactions by assuming that the transition state (TS) of analogous chemical reactions represents the transition state (TS) of the electrochemical reaction. This assumption allows us to evaluate the intrinsic activity of catalysts towards (R1) and (R2) without applying a potential. (R3) is originally a chemical (non-electrochemical) reaction that is independent of potential. ${ }^{38}$ Fig. 7 shows the simulation results of the Volmer reaction on $1 \mathrm{~T}-\mathrm{MoS}_{2}, 2 \mathrm{H}-\mathrm{MoS}_{2}$, and at different sites on the $1 \mathrm{~T} / 2 \mathrm{H}$ interface. The barrier energy of all catalysts is about $0.90 \mathrm{eV}$, which originates from the dissociation of $\mathrm{H}_{3} \mathrm{O}^{+}$. The similar barrier energy of all catalysts suggests that catalysts contribute little to the dissociation, and this process will be completed by the applied potential. The total energy of the final product could represent the stability after catalyst binding with $\mathrm{H}^{*}$, which could be used to compare the binding abilities. $1 \mathrm{~T}-\mathrm{MoS}_{2}$ has a reaction energy of $-0.76 \mathrm{eV}$, which is almost twice that of $2 \mathrm{H}-\mathrm{MoS}_{2}(-0.43 \mathrm{eV})$. The high reaction energy of $1 \mathrm{~T}-\mathrm{MoS}_{2}$ indicates that it has a better binding ability

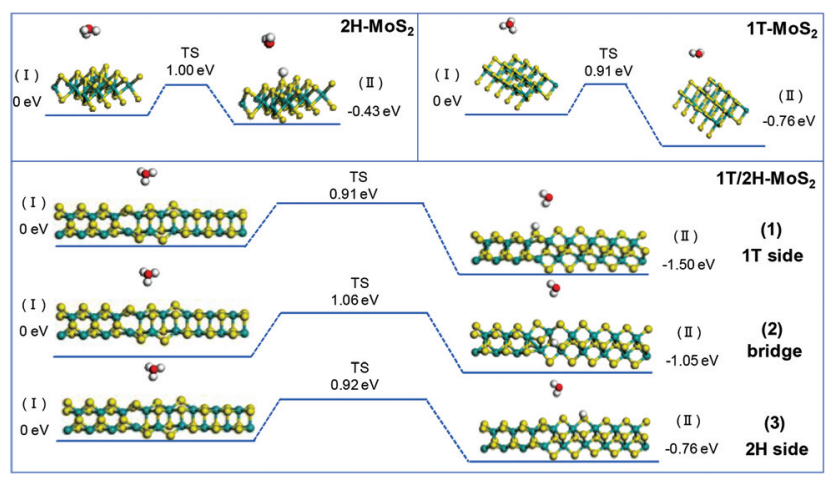

Fig. 7 Geometric structures of the initial state (I) and final state (II) of the Volmer reaction and its energetics on (100)-S atoms of $1 \mathrm{~T}-\mathrm{MoS}_{2}$ and $1 \mathrm{~T} / 2 \mathrm{H}$ interface. 
than $2 \mathrm{H}-\mathrm{MoS}_{2}$. Interestingly, the reaction energy on the $1 \mathrm{~T} / 2 \mathrm{H}$ interface is higher than or similar to that on $1 \mathrm{~T}-\mathrm{MoS}_{2}$, which indicates that the distorted structure could activate the $\mathrm{S}$ atom near the interface. Owing to the activation, the $\mathrm{S}$ atom close to the $1 \mathrm{~T}$ side exhibits the highest reaction energy $(-1.50 \mathrm{eV})$. Besides, the $\mathrm{S}$ atom close to the $2 \mathrm{H}$ side exhibits similar reaction energy to $1 \mathrm{~T}-\mathrm{MoS}_{2}(-0.76 \mathrm{eV}$ vs. $-0.76 \mathrm{eV})$.

The simulation of Heyrovsky reactions (R2) is shown in Fig. 8. The barrier energy in this step represents the energy that surface $\mathrm{H}^{*}$ desorption process under the effect of catalysts, and the dissociation of another $\mathrm{H}_{3} \mathrm{O}^{+}$. In this step, $2 \mathrm{H}-\mathrm{MoS}_{2}$ and $1 \mathrm{~T}-\mathrm{MoS}_{2}$ show similar activities (1.64 eV vs. 1.71 $\mathrm{eV}$ ) on the (100) S sites. On the interface of $1 \mathrm{~T} / 2 \mathrm{H}-\mathrm{MoS}_{2}$, the barrier height is quite different. The $S$ atom close to the $1 \mathrm{~T}$ side shows a much lower barrier $(0.83 \mathrm{eV})$ than $1 \mathrm{~T}$ or $2 \mathrm{H}-\mathrm{MoS}_{2}$, which indicates its much higher activity in the Heyrovsky reaction. However, the energy barrier on the bridge $\mathrm{S}$ atom and $2 \mathrm{H}$ side $\mathrm{S}$ atom is much higher than pure $1 \mathrm{~T}$ or $2 \mathrm{H}-\mathrm{MoS}_{2}$, which means they are relatively unfavorable for the Heyrovsky reaction. Based on the above analysis, the activity of VolmerHeyrovsky process on the (100) surface could be concluded as follows: (1) the $2 \mathrm{H}-\mathrm{MoS}_{2}$ sample exhibits the worst performance in the overall Volmer-Heyrovsky process; (2) the $1 \mathrm{~T} / 2 \mathrm{H}$ interfacial $\mathrm{S}$ atom near the $1 \mathrm{~T}$ side exhibits the best simulatedactivity in the overall Volmer-Heyrovsky process which is even better than a pure 1T- $\mathrm{MoS}_{2}$ sample; (3) other $1 \mathrm{~T} / 2 \mathrm{H}$ interfacial sites exhibit a better performance in the Volmer step, but worse performance in the Heyrovsky process than $1 \mathrm{~T}-\mathrm{MoS}_{2}$ which could hardly contribute to the overall Volmer-Heyrovsky process. Another process, the Volmer-Tafel process, was also simulated under four patterns of neighboring interfacial $\mathrm{S}$ sites together with $1 \mathrm{~T}-\mathrm{MoS}_{2}$. The results are shown in Fig. S11. $\dagger$ It should be noted that the model of $2 \mathrm{H}-\mathrm{MoS}_{2}$ with two adsorbed $\mathrm{H}^{*}$ could not be stabilized by geometry optimization, suggesting that the Volmer-Tafel process does not occur on the (100) neighboring S sites of $2 \mathrm{H}-\mathrm{MoS}_{2}$. The simulation results of the Tafel reaction all show high barrier energy (above $2.5 \mathrm{eV}$ ), which indicates that the reaction is unfavorable on the catalysts. Among different adsorbed sites on the interface, $2 \mathrm{H}-$

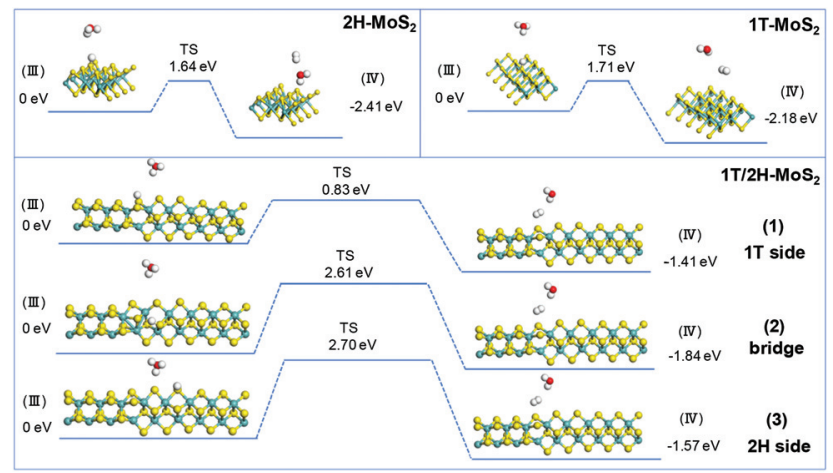

Fig. 8 Geometric structures of the initial state (III) and final state (IV) of the Heryvosky reaction and its energetics on (100)-S atoms of $1 \mathrm{~T}-\mathrm{MoS}_{2}$ and $1 \mathrm{~T} / 2 \mathrm{H}$ interface. bridge sites exhibit a reaction energy of $0.02 \mathrm{eV}$, which indicates that the reaction is even endothermic. Considering that the Tafel reaction is impossible on $2 \mathrm{H}-\mathrm{MoS}_{2}$, the reaction is still exothermic on the other patterns of interfacial sites, which suggests the least possibility of the Tafel reaction. However, compared with the Volmer-Heryvosky process, the Volmer-Tafel process on $\mathrm{MoS}_{2}$ exhibits higher barrier energy and smaller total Gibbs free energy, which indicates that the main path of the HER catalyzed by $\mathrm{MoS}_{2}$ is the VolmerHeyrovsky mechanism. In conclusion, $1 \mathrm{~T}-\mathrm{MoS}_{2}$ exhibits a better activity than $2 \mathrm{H}-\mathrm{MoS}_{2}$. On the interface of $1 \mathrm{~T} / 2 \mathrm{H}-\mathrm{MoS}_{2}$, the interfacial $\mathrm{S}$ atoms show even better $\mathrm{H}^{*}$ adsorption ability than active $1 \mathrm{~T}-\mathrm{MoS}_{2}$, which demonstrates the activation of the interfacial S atoms by the distortion of the structure. The interfacial $\mathrm{S}$ atoms near the $1 \mathrm{~T}$ side exhibit the best overall VolmerHeyrovsky reaction activity among $1 \mathrm{~T}-\mathrm{MoS}_{2}, 2 \mathrm{H}-\mathrm{MoS}_{2}$, and other sites on the interface. As the conclusion, the HER performance is positively correlated with the $1 \mathrm{~T}$ concentration, and the HER performance will also be affected by the amount of $1 \mathrm{~T} / 2 \mathrm{H}$ interface.

\section{Conclusions}

In conclusion, hybrid $1 \mathrm{~T} / 2 \mathrm{H}-\mathrm{MoS}_{2}$ was synthesized and the $1 \mathrm{~T}$ concentration was controlled by using different precursors. In a solvothermal method using DMF, the $1 \mathrm{~T}$ content could be controlled by the different proportions of water and DMF. The highest $1 \mathrm{~T}$ concentration reached was $70.13 \%$. Another hydrothermal synthesis with a guest ion agent $\left(\mathrm{NH}_{4} \mathrm{HCO}_{3}\right)$ could also create $1 \mathrm{~T} / 2 \mathrm{H}-\mathrm{MoS}_{2}$ with a $1 \mathrm{~T}$ concentration of around $60 \%$. In this method, the fixed $1 \mathrm{~T}$ content hybrid phase $\mathrm{MoS}_{2}$ could be synthesized using a wide range of precursor amount, and $60 \% 1 \mathrm{~T}$ content $1 \mathrm{~T} / 2 \mathrm{H}-\mathrm{MoS}_{2}$ could be stably synthesized. The hydrogen evolution reaction performance was positively correlated with the $1 \mathrm{~T}$ concentration. By introducing a higher content of the $1 \mathrm{~T}$ phase, $\mathrm{MoS}_{2}$ could act as highly-active HER catalysts, which was also confirmed by DFT calculations on the (100) surface of $1 \mathrm{~T}-\mathrm{MoS}_{2}, 2 \mathrm{H}-\mathrm{MoS}_{2}$ and $1 \mathrm{~T} / 2 \mathrm{H}$ interface. This work provides a scientific basis for applying hybrid phase $\mathrm{MoS}_{2}$ in the electrochemical hydrogen evolution reaction.

\section{Conflicts of interest}

There are no conflicts to declare.

\section{Acknowledgements}

This work was supported by the International Joint Research Promotion Program of Osaka University. The present work was also supported by the Grant-in-Aid for Scientific Research from the Ministry of Education, Culture, Sports, Science and Technology (MEXT) of Japan (no. 19H008380). Part of this work was supported by Element Strategy Initiative of MEXT, Japan (no. JPMXP0112101003). The synchrotron radiation 
experiments for XAFS measurements were performed at the BL01B1 beamline in SPring-8 with the approval of JASRI (no. 2018B1082 and 2018B1185).

\section{References}

1 M. A. Lukowski, A. S. Daniel, F. Meng, A. Forticaux, L. Li and S. Jin, J. Am. Chem. Soc., 2013, 135, 10274-10277.

2 D. Voiry, M. Salehi, R. Silva, T. Fujita, M. Chen, T. Asefa, V. B. Shenoy, G. Eda and M. Chhowalla, Nano Lett., 2013, 13, 6222-6227.

3 Y. Zhang, Y. Kuwahara, K. Mori and H. Yamashita, Chem. Asian J., 2019, 14, 278-285.

4 Y. Zhang, P. Ju, C. Zhao and X. Qian, Electrochim. Acta, 2016, 219, 693-700.

5 C. Zhao, Y. Zhang and X. Qian, Electrochim. Acta, 2016, 198, 135-143.

6 U. Maitra, U. Gupta, M. De, R. Datta, A. Govindaraj and C. N. R. Rao, Angew. Chem., Int. Ed., 2013, 52, 13057-13061.

7 A. B. Laursen, S. Kegnæs, S. Dahl and I. Chorkendorff, Energy Environ. Sci., 2012, 5, 5577.

8 Y. Li, H. Wang, L. Xie, Y. Liang, G. Hong and H. Dai, J. Am. Chem. Soc., 2011, 133, 7296-7299.

9 X. Lin, D. Xue, L. Zhao, F. Zong, X. Duan, X. Pan, J. Zhang and Q. Li, Chem. Eng. J., 2019, 356, 483-491.

10 K. Chang, X. Hai, H. Zhang, S. Li, G. Liu, H. Liu, G. Zhao, M. Li and J. Ye, Adv. Mater., 2016, 28, 10033-10041.

11 T. Xiang, Q. Fang, H. Xie, C. Wu, C. Wang, Y. Zhou, D. Liu, S. Chen, A. Khalil, S. Tao, Q. Liu and L. Song, Nanoscale, 2017, 9, 6975-6983.

12 X. Geng, Y. Jiao, Y. Han, A. Mukhopadhyay, L. Yang and H. Zhu, Adv. Funct. Mater., 2017, 27, 1702998.

13 F. Wypych and R. Schöllhorn, J. Chem. Soc., Chem. Commun., 1992, 1386-1388.

14 R. Miao, B. Dutta, S. Sahoo, J. He, W. Zhong, S. A. Cetegen, T. Jiang, S. P. Alpay and S. L. Suib, J. Am. Chem. Soc., 2017, 139, 13604-13607.

15 W. Lubitz and W. Tumas, Chem. Rev., 2007, 107, 3900-3903.

16 Z. Cao, Q. Chen, J. Zhang, H. Li, Y. Jiang, S. Shen, G. Fu, B. Lu, Z. Xie and L. Zheng, Nat. Commun., 2017, 8, 15131.

17 Q. Tang and D. Jiang, ACS Catal., 2016, 6, 4953-4961.

18 M. A. Lukowski, A. S. Daniel, F. Meng, A. Forticaux, L. Li and S. Jin, J. Am. Chem. Soc., 2013, 135, 10274-10277.
19 Y. Jiao, A. M. Hafez, D. Cao, A. Mukhopadhyay, Y. Ma and H. Zhu, Small, 2018, 14, 1800640.

20 S. Jayabal, G. Saranya, J. Wu, Y. Liu, D. Geng and X. Meng, J. Mater. Chem. A, 2017, 5, 24540-24563.

21 S. Wang, D. Zhang, B. Li, C. Zhang, Z. Du, H. Yin, X. Bi and S. Yang, Adv. Energy Mater., 2018, 8, 1801345.

22 J. Yang, K. Wang, J. Zhu, C. Zhang and T. Liu, ACS Appl. Mater. Interfaces, 2016, 8, 31702-31708.

23 L. Wang, Z. Xu, W. Wang and X. Bai, J. Am. Chem. Soc., 2014, 136, 6693-6697.

24 Q. Huang, X. Li, M. Sun, L. Zhang, C. Song, L. Zhu, P. Chen, Z. Xu, W. Wang and X. Bai, Adv. Mater. Interfaces, 2017, 4, 1700171.

25 Z. Wu, C. Tang, P. Zhou, Z. Liu, Y. Xu, D. Wang and B. Fang, J. Mater. Chem. A, 2015, 3, 13050-13056.

26 D. Wang, Y. Xiao, X. Luo, Z. Wu, Y. Wang and B. Fang, ACS Sustainable Chem. Eng., 2017, 5, 2509-2515.

27 Y. Zhang, Y. Kuwahara, K. Mori and H. Yamashita, Langmuir, 2020, 36, 1174-1182.

28 Y. Liu, Y. Li, F. Peng, Y. Lin, S. Yang, S. Zhang, H. Wang, Y. Cao and H. Yu, Appl. Catal., B, 2019, 241, 236-245.

29 H. Fan, R. Wu, H. Liu, X. Yang, Y. Sun and C. Chen, J. Mater. Sci., 2018, 53, 10302-10312.

30 L. Cai, J. He, Q. Liu, T. Yao, L. Chen, W. Yan, F. Hu, Y. Jiang, Y. Zhao, T. Hu, Z. Sun and S. Wei, J. Am. Chem. Soc., 2015, 137, 2622-2627.

31 T. Yoshii, K. Nakatsuka, Y. Kuwahara, K. Mori and H. Yamashita, Chem. Lett., 2017, 46, 789-791.

32 K. Nakatsuka, T. Yoshii, Y. Kuwahara, K. Mori and H. Yamashita, Phys. Chem. Chem. Phys., 2017, 19, 49674974.

33 D. Wang, X. Zhang, S. Bao, Z. Zhang, H. Fei and Z. Wu, J. Mater. Chem. A, 2017, 5, 2681-2688.

34 M. Acerce, D. Voiry and M. Chhowalla, Nat. Nanotechnol., 2015, 10, 313.

35 G. Eda, H. Yamaguchi, D. Voiry, T. Fujita, M. Chen and M. Chhowalla, Nano Lett., 2011, 11, 5111-5116.

36 D. Ghim, Q. Jiang, S. Cao, S. Singamaneni and Y.-S. Jun, Nano Energy, 2018, 53, 949-957.

37 Y. Zhang, Z. Mu, C. Yang, Z. Xu, S. Zhang, X. Zhang, Y. Li, J. Lai, Z. Sun, Y. Yang, Y. Chao, C. Li, X. Ge, W. Yang and S. Guo, Adv. Funct. Mater., 2018, 28, 1707578.

38 W. Li, G. Liu, J. Li, Y. Wang, L. Ricardez-Sandoval, Y. Zhang and Z. Zhang, Appl. Surf. Sci., 2019, 498, 143869. 